\title{
Stimulus-induced rhythmic, periodic, or ictal discharges (SIRPIDs): an intriguing EEG phenomenon
}

\author{
Descargas ictais, periódicas ou rítmicas induzidas por estímulos (SIRPIDs): \\ um fenômeno EEG intrigante
}

Mariana Ribeiro Marcondes da Silveira' , Joaquina Andrade², Eliana Garzon ${ }^{3}$

\begin{abstract}
:
SIRPIDs, an acronym for stimulus-induced rhythmic, periodic, or ictal discharges, were first named in 2004. This is a pattern observed in continuous electroencephalogram (CEEG) consistently elicited by stimulation in comatose patients. The pathophysiology of SIRPIDs probably involves dysregulation of subcortico-cortical projections, particularly thalamocortical circuit, in a markedly abnormal brain with hyperexcitable cortex. This may explain some studies found an association of prolonged periodic epileptiform discharges (PEDs) activity and a higher incidence of concurrent electrographic seizures and SIRPIDs. An association of SIRPIDs and poor prognosis has already been described. However, it is not yet possible to assert whether these discharges can cause neuronal injury or if they are simply a marker of severe brain injury. Objective of this paper is to review clinical relevance and pathophysiology of SIRPIDs, as well as its role as a brain response in the critically ill patient.
\end{abstract}

Keywords: SIRPIDs, PEDs, electroencephalography, comatose patient, continuous video electroencephalography, nonconvulsive status epilepticus.

\section{RESUMO:}

O termo SIRPIDs é um acrônimo do inglês que pode ser traduzido como: descargas ictais, periódicas ou rítmicas induzidas por estímulos e foi utilizado pela primeira vez em 2004. Este padrão é observado no eletroencefalograma contínuo (CEEG) obtido pela estimulação de pacientes comatosos. A fisiopatologia dos SIRPIDs provavelmente envolve uma falha na regulação das projeções corticais e subcorticais, particularmente nos circuitos talamocorticais, num cérebro anormal com o córtex hiperexcitável. Isso pode explicar a associação encontrada por alguns estudos entre as descargas epileptiformes periódicas (PEDs) prolongadas e uma maior incidência de crises eletrográficas, bem como de SIRPIDs. É descrita associação entre os SIRPIDs e pior prognóstico. Ainda não é possível determinar se estas descargas podem causar dano neuronal ou se elas são simplesmente marcadoras de lesão cerebral grave. O objetivo deste artigo é revisar a relevância clínica, a fisiopatologia dos SIRPIDs e seu papel como resposta cerebral no paciente crítico.

Palavras-chave: descargas ictais, periódicas ou rítmicas induzidas por estímulos; descarga epileptiforme periódica; paciente comatoso; videoeletroencefalograma contínuo; status epilepticus não convulsivo.

Continuous electroencephalography (CEEG) is recommended whenever early detection of a pathophysiological process may prevent its long-lasting consequences. CEEG has been increasingly carried out in intensive care units all over the world, playing an important role in the management of unconscious patient ${ }^{1,2}$.
In 2004, using CEEG, an intriguing phenomenon recorded on electroencephalogram (EEG) and called SIRPIDs, an acronym for stimulus-induced rhythmic, periodic, or ictal discharges, was described ${ }^{3}$. This pattern was consistently elicited by stimulation, and was first observed accidentally, during the examination of stuporous or comatose patients ${ }^{2,3}$.

${ }^{1}$ MD, Staff da Seção de Eletroencefalografia, Divisão de Neurologia, Hospital das Clínicas da Faculdade de Medicina da Universidade de São Paulo, Sao Paulo SP, Brazil;

${ }^{2}$ MD, Staff da Seção de Eletroencefalografia, Divisão de Neurologia, Hospital das Clínicas da Faculdade de Medicina da Universidade de São Paulo, Sao Paulo SP, Brazil;

${ }^{3} \mathrm{MD}, \mathrm{PhD}$, Coordinator da Seção de Eletroencefalografia, Divisão de Neurologia, Hospital das Clínicas da Faculdade de Medicina da Universidade de São Paulo, Sao Paulo SP, Brazil.

Correspondence: Mariana Ribeiro Marcondes da Silveira; Avenida Doutor Enéas de Carvalho Aguiar 255 / PAMB / 6 Andar / Bloco 4; $05403-900$ São Paulo SP - Brasil; E-mail: ribeiromarcondes7@gmail.com

Conflict of interest: There is no conflict of interest to declare.

Received 24 June 2013; Received in final form 18 July 2013; Accepted 25 July 2013. 
The pace of technological advances that makes CEEG recording possible allows the acknowledgement of SIRPIDs and other controversial EEG patterns, such as periodic ones. However, the comprehension of their pathophysiological process has lagged behind ${ }^{4}$. Not understanding the pathophysiology of these EEG patterns raises a series of interesting questions, including: are these discharges causing neuronal injury or are they only a marker of severe brain injury? Are they contributing to the patient's abnormal mental status or do they have a specific prognostic meaning? Will pharmacologically suppressing these discharges help these patients, or modify their follow-up ${ }^{3}$ ?

The review of certain concepts in the current literature including definition of terminology, pathophysiology, clinical relevance and its role as brain response in critically ill patients were the main goals of this paper.

\section{METHOD}

All papers referring to SIRPIDs were included. The papers were found with SIRPIDs as a key-word at PubMed. Additional papers to make clear definitions or for better reflection about the pathophysiological process were also included in a non systematic review.

\section{Terminology}

In the literature, terms were defined according EEG definitions from the American Clinical Neurophysiology Society terminology of periodic and rhythmic EEG patterns in the critically ill for research purposes ${ }^{5}$.
SIRPIDs: were defined as periodic, rhythmic, or ictal appearing discharges that were consistently induced by alerting stimuli such as auditory stimuli, sternal rub, examination, suctioning, turning, and other patient-care activities (Figure). They may also occur spontaneously, or with an unnoticeable or even internal alerting stimulus. A testable alerting stimulus must, by definition, be able to reproduce the response $\mathrm{e}^{2,3}$.

The duration of an individual episode of SIRPIDs, as previously described, ranged from seconds to hours, but most commonly for a few minutes and was highly variable in many patients. The type of stimulation required to elicit SIRPIDs was variable. Any noise was enough in some patients, whereas noxious stimulation was required in others ${ }^{3}$.

Periodic: pattern consisted of discharges (sharp waves, spikes, polyspikes, or sharply contoured delta waves) recurring at regular or nearly regular intervals with an identifiable interdischarge interval ${ }^{3}$.

Rhythmic: when the pattern became continuous with no separation between individual discharges, the pattern was then considered rhythmic rather than periodic ${ }^{3}$.

Ictal-appearing discharge: any rhythmic discharge or spike-and-wave pattern with definite evolution in frequency, location, or morphology. Evolution in amplitude alone did not qualify a record as ictal-appearing ${ }^{3}$.

\section{The beginning}

A small number of references had reported stimulusinduced discharges before 2004. The first description was a consistent increase in the amplitude of periodic epileptiform lateralized discharges (PLEDs) as an effect of painful stimuli 6 . The same paper also describes a patient who had numerous

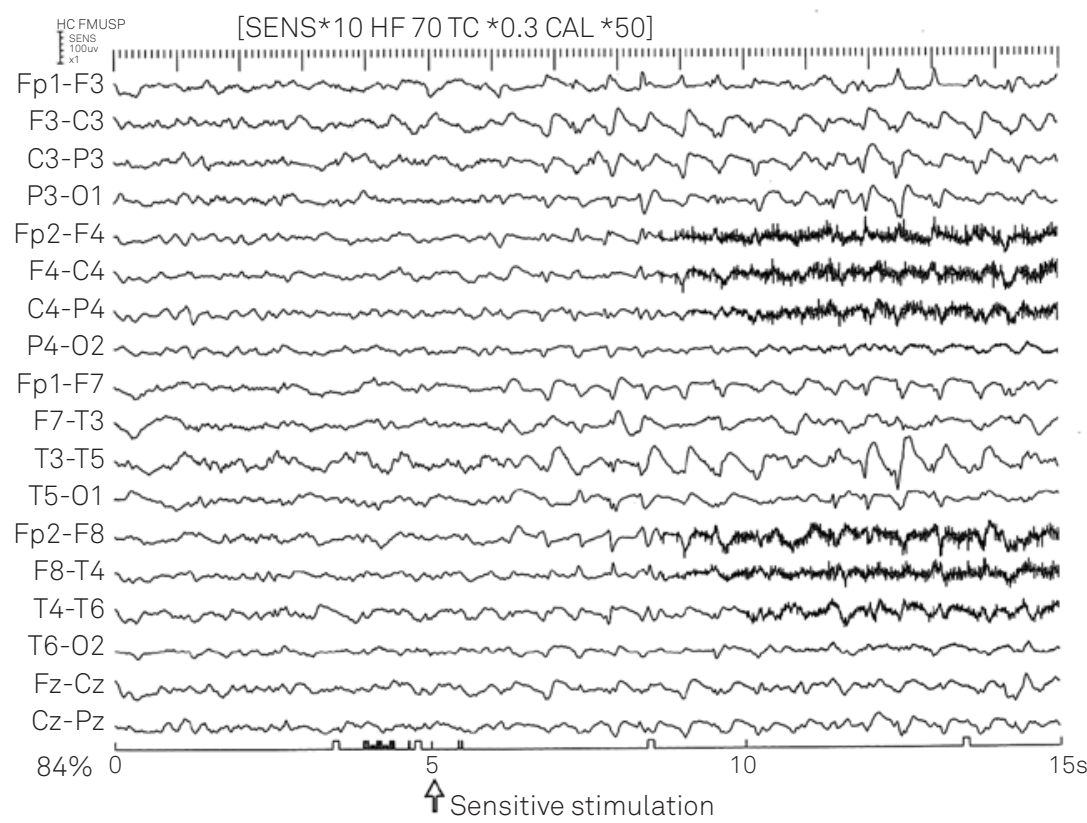

Figure. 51-years-old female patient. Previous history of focal epilepsy. Nonconvulsive status epilepticus achieved control with continuous Midazolam. Periodic sharp and slow waves over the left side following sensitive stimulation. Muscle artifacts over the right side. 
seizure discharges, which were induced by painful stimuli with no apparent clinical changes.

It is believed that alerting stimuli might obtain triphasic waves more prominent in patients with metabolic disorders and trifasic waves that are less prominent in deep coma. Moreover, studies which specifically addressed this issue found no consistent EEG change during stimulation in patients with triphasic waves?

Before the identification of SIRPIDs ${ }^{3}$, a similar feature was observed and reported under a slightly different acronym ${ }^{8}$. The acronym SIRPIDs (stimulus-responsive pseudoictal discharges) was used for describing EEG changes due to external stimulus in critically ill patients.

SIRPIDs, as a term, came about two years later in a study of a larger group of patients. After review 150 consecutive patients undergoing CEEG, the authors identified 33 with SIRPIDs (22\%). The majority of these patients (73\%) had acute brain injuries while only eight patients had a prior history of epilepsy.

In 21 patients the EEG pattern, induced by alerting stimuli, consisted of periodic epileptiform discharges (nine lateralized). In 18, there were rhythmic patterns with evolution that fulfilled the criteria for ictal discharges (12 unilateral). No correlation was found between different subtypes of SIRPIDs and clinical findings.

Half the patients (17 of 33) had unequivocal seizures at some point during their acute illness. Of the patients with seizures, ten had clinically overt seizures, whereas seven had subclinical or subtle seizures and only one had subclinical and overt clinical seizures. Status epilepticus (SE) occurred in 11 patients. All patients were stuporous or comatose on the day of their EEG showing SIRPIDs. Although half of patients had subclinical or overt seizures, and 11 had SE, no clinical correlation to SIRPIDs was seen in any patient except in one who had irregular jerking in one arm during stimulation-induced generalized periodic epileptiform discharges (GPEDs).

The comparison between the group with and without SIRPIDs showed no significant difference. The incidence of clinical seizures in patients with SIRPIDs was $45 \%$ compared to $30 \%$ in those without SIRPIDs.

Clinical SE occurred in $24 \%$ of patients with SIRPIDs and in $17 \%$ of those without SIRPIDs. Clinical SE was not associated with SIRPIDs overall, but was independently associated with both ictal-appearing and focal SIRPIDs on multivariate analysis.

The conclusion of this retrospective study was an unclear relationship between clinical seizures and SIRPIDs.

The same researchers brought up again for discussion, the relationship between seizures and SIRPIDs in $2008^{2}$. At this time, nine patients, all of them comatose, had focal motor seizures, repeatedly elicited by alerting stimuli. Eight patients had nonconvulsive SE at some point of their acute illness. The conclusion now was that alerting stimuli can induce seizures in encephalopathic/comatose patients. For the authors, the presence of clinical seizures induced by alerting means that at least some of these stimulus induced electrographic events are indeed seizures, and not simply an abnormal arousal or other process not directly related to seizures ${ }^{2}$.

\section{Reflections on pathophysiology}

The pathophysiology of SIRPIDs is not known but it is supposed that the occurrence of SIRPIDs probably involves dysregulation of subcortico-cortical projections, particularly thalamocortical circuitry, in a markedly abnormal brain with hyperexcitable cortex ${ }^{3}$. Metabolic abnormalities appear to play an important role as well ${ }^{6}$.

Arousal begins in the upper brainstem, especially in the midbrain reticular formation, and it is mediated by a complex circuit involving reciprocal connections between cortex and thalamus ${ }^{9,10}$. Experiments have demonstrated that cortical projections can affect intrinsic thalamic oscillations and the behavioral state is determined by oscillations involving thalamus ${ }^{11}$. The thamocortical projections are also involved in some seizures ${ }^{10}$.

The conclusion about the pathophysiology of SIRPIDs is that if arousal is directly involved with thalamic neurons with intrinsic oscillation properties, it is possible that the activation of this circuit in an abnormal situation could elicit rhythmic or periodic discharges ${ }^{3}$.

This assumption requires caution, as the true mechanism underlying periodic patterns is still under research.

Hypnopompic hypersynchrony (high-voltage rhythmic delta activity during normal arousal in children), and the abnormal frontal rhythmic delta seen on arousal in encephalopathic adults, named "paradoxical arousal" had been compared in terms of pathophysiological explanation to the abnormal periodic and rhythmic patterns related to arousal ${ }^{3}$.

\section{An entity to be understood and looked for}

Since SIRPIDs' description, several case reports brought a great deal of interesting examples showing the need for further systematic studies in this field.

One example is the case of an unresponsive 50 year-old woman with a history of coronary artery bypass, drug addiction and Diabetes Mellitus, presenting a brain stem infarction and SIRPIDs in her first EEG record. She had two different SIRPIDs patterns, periodic and rhythmic ${ }^{12}$.

SIRPIDs elicited by sensitive stimulation of restricted and specific face area were described in one patient, 39 year-old cocaine addict and postanoxic encephalopathy after prolonged cardiac resuscitation ${ }^{13}$. In this case, EEG showed a burstsuppression pattern with generalized spike-wave activity in the burst phases. This activity consistently started after touching exclusively the area of the patient's forehead and eyelids and was clinically accompanied by bilateral periorbicular twitching. 
The intriguing point is that SIRPIDs were exclusively induced by tactile stimulation of the area innervated by the ophthalmic nerve, which had not been described until then. The authors discussed whether this peculiar case could be justified by the neurological situation of tetraplegy ${ }^{13}$.

Sporadic Creutzfeldt-Jakob disease (CJD) of a 74 yearold woman with a history of a rapidly worsening progressive mental status over the previous three weeks, was reported to have SIRPIDs with a periodic pattern ${ }^{14}$. During the initial phase of the disease, EEG presented disorganized alpha-theta background and, paralleling the changes in responsiveness, intermittently superimposed irregular spikes (about 2-3 Hz), non-reactive, slightly predominating on the right side. Her initial diagnosis was nonconvulsive SE. Since of no clinical and electrographical responses were obtained with clonazepan and phenytoin, propofol was given to her. Three hours after weaning her of propofol, SIRPIDs with periodic pattern were observed. Later, her brain MRI and determination CSF 14-3-3-b protein suggested diagnosis of sporadic CJD which was pathologically confirmed after her death. The occurrence of SIRPIDs and an unusual presentation case of CJD deserves more discussion ${ }^{14}$.

More interesting was the report of a 63-year-old man with viral encephalitis that had SIRPIDs on his EEG. SIRPIDs pattern was bi-central periodic epileptiform discharges. This electrographic pattern was associated with myoclonus when provoked by strong and protracted stimuli, although it remained subclinical when elicited by auditory or mild tactile stimuli. Spontaneous and stimulus-induced bi-central PEDs disappeared after full neurological recovery of this patient ${ }^{15}$.

In an attempt to better understand PEDs in comatose patients and its relationship to follow-up, comatose patients who underwent CEEG lasting ten or more consecutive days, were retrospectively studied according presence and duration of PEDs ${ }^{16}$. Sixty seven patients, mean age of 56 years (range 18-83 yrs), could be classified as prolonged PEDs (PEDs for at least five days); intermittent PEDs (at least one but fewer than five consecutive days with PEDs), or with no PEDs $^{16}$. The most common diagnoses of these patients were hypoxia ischemic encephalopathy, subarachnoid hemorrhage, epilepsy, encephalitis, metabolic encephalopathy and intracerebral hemorrhage. SIRPIDs were found in $34 \%$ of total study population.

Although prolonged PEDs were associated with electrographic seizures and SIRPIDs, they had no impact on mortality, which was $31 \%$ overall. Prolonged PEDs also had no association with recovery of consciousness at the time of discharge, which was $36 \%$ overall $^{16}$.

Usually in critically ill patients, SIRPIDs have no clinical correlation. Spontaneous seizures in comatose patients can also be under-recognized and CEEG is necessary in detecting seizures ${ }^{17}$. Seizures were detected in $19 \%$ of a group of 570 consecutive patients who underwent CEEG monitoring for the detection of subclinical seizures or evaluation of unexplained decrease in levels of consciousness ${ }^{17}$. The seizures were exclusively nonconvulsive in $92 \%$ of these patients ${ }^{17}$. Nonconvulsive seizures and SIRPIDs should be looked for, since they can be underestimated in clinical practice.

\section{Abnormal arousal, ictal or lesional pattern?}

The discussion about the meaning of SIRPIDs continues.

To determine if SIRPIDs represent an ictal phenomenon, a case was investigated with cerebral perfusion on singlephoton emission computerized tomography (SPECT). If SIRPIDs correspond to an ictal pattern, an increase of cerebral perfusion is expected.

A 62 year-old female patient with occlusion of the left internal carotid artery at its origin, from the bifurcation, and nonconvulsive SE, confirmed by EEG recording evolved with SIRPIDs (rhythmic 2- to 3-Hz delta activity). To test the ictalincreased flow hypothesis, a SPECT was obtained during a stimulus-induced train of rhythmic discharges on EEG. The SPECT revealed no increase in tracer uptake ${ }^{18}$.

Some authors suggest that regional cerebral blood flow ( $\mathrm{rCBF}$ ) sensitivity for seizure activity is as high as 94\%; but the nature of SIRPIDs, if it is ictal or inter-ictal remains somewhat unclear.

One explanation for this conclusion is that $\mathrm{rCBF}$ is not necessarily a marker of active seizures per se but of increased neuronal activity that can occur in other clinical conditions besides seizures. From another point of view, a negative SPECT argues against ongoing seizure activity ${ }^{18}$.

For testing the lesional meaning of SIRPIDs, a prospective study in Intensive Care Unit after cardiac arrest treated with therapeutic hypothermia enrolled 144 patients with 105, having EEG during both therapeutic hypothermia and normothermia ${ }^{19}$. The mean age was 61.1 years $(+/-12.8)$ and the prevalence of SIRPIDs was $13.3 \%$ (14/105 patients) in this study (19). Eight of them had a periodic pattern, while the others were equally distributed between rhythmic or ictal patterns. Elevated serum neuron-specific enolase was more associated in patients with SIRPIDs $(62 \%)$ than in patients without SIRPIDs (29\%).

Overall, SIRPIDs were associated with poor prognosis at three months, particularly when occurring during therapeutic hypothermia ${ }^{19}$. SIRPIDs were also associated with discontinuous or non-reactive EEG background and were robustly related to poor neurological outcome ${ }^{19}$.

Survival and favorable functional outcome, however, may be observed when SIRPIDs appeared after rewarming and in normothermic conditions ${ }^{19}$.

The correlation of SIRPIDs with elevated neuron-specific enolase could suggest SIRPIDs as a lesional pattern rather than an ictal one ${ }^{19}$.

It is interesting that there is a report describing a patient with GPEDs which were consistently "abolished" by alerting stimuli ${ }^{20}$. 
An EEG of a 70-year-old woman with pneumococcal meningoencephalitis showed runs of epileptiform discharges characterized by diffuse spike-wave complex occurring every 1-2 s which consistently disappeared for 2-7 s after nociceptive and acoustic stimulations ${ }^{20}$. The findings were interpreted as a sign of reactivity of the EEG rhythms and thus as a possibly favorable sign ${ }^{20}$. The authors hypothesized that, similarly to SIRPIDs, the pathophysiology of stimulus abolished PEDs probably involves dysregulation of thalamocortical and other cortical-subcortical projections in a brain with hyperexcitable cortex ${ }^{20}$.

In conclusion, SIRPIDs are an electrographical pattern recently described, that occur during the examination of the stuporous or comatose patient.

The pathophysiology of SIRPIDs probably involves dysregulation of subcortico-cortical projections, particularly thalamocortical circuit, in a markedly abnormal brain with hyperexcitable cortex ${ }^{3}$.

There is one study showing association of SIRPIDs and poor prognosis (19). However, it is not yet possible to say that these discharges can cause neuronal injury or if they are simply a marker of severity of brain injury.

We know that important questions remain unanswered especially about the treatment. The best answer for now is that nobody knows exactly and larger scale studies need to be performed.

Studies including SPECT would be ideal for answering these questions. They must be carried out with other patterns of SIRPIDs, such as ictal-appearing or even PEDs or any other examples of delta rhythmic, because perhaps SPECT results would be different for each pattern.
Prolonged PEDs were correlated to SIRPIDs and electrographic seizures ${ }^{16}$. The evidences for or against SIRPIDs as an ictal pattern are few and contradictory.

There is no formal standard of care for PEDs which have been known for a much long time than SIRPIDs. What is done for PEDs is a reasonable approach, including an investigation into the cause, sometimes, initiation of conventional antiepileptic drugs (AEDs) for prophylaxis for seizures, and to continue EEG monitoring for recognizing nonconvulsive seizures or $\mathrm{SE}^{4}$. This is not a formal recommendation, but it is a reasonable one for the patient with SIRPIDs too, except maybe for initiation of AEDs, that has to be evaluated clinically in each case. This is because the correlation of SIRPIDs and ictal patterns are poorer than PEDs and PLEDs.

An interesting recommendation for day-to-day on treatment of nonconvulsive SE and coma, published in 2010, would help us ${ }^{21}$. A complete classification based on clinical and EEG aspects must always consider depth of coma and the degree of structural brain damage and epileptic brain dysfunction. Next, one should try to understand whether if the coma is caused by the EEG findings or the brain disorder. If eventual epileptic activity could contribute to the depth of coma and worsen the prognosis, then you should treat all forms of epileptic activity. The prescription of parenteral AEDs should be done with caution because of their morbidity, among other things, their risk of hypotension and respiratory suppression ${ }^{18,21}$.

In spite of a lack of answers using this rationale with SIRPIDs would be useful guide for clinicians and future studies.

There is a long way so before we really understand this fascinating form of hyperexcitable cortex responding to a stimulus and what message the brain is trying to send us.

\section{References}

1. Guérit JM, Amantini A, Amodio P, et al. Consensus on the use of neurophysiological tests in the intensive care unit (ICU): electroencephalogram (EEG), evoked potentials (EP), and electroneuromyography (ENMG). Neurophysiol Clin 2009;39:71-83.

2. Hirsch JL, Pang T, Claassen J, et al. Focal motor seizures induced by alerting stimuli in critically ill patients. Epilepsia 2008;49:968-973.

3. Hirsch LJ, Claassen J, Mayer SA, Emerson RG. Stimulus-induced rhythmic, periodic, or ictal discharges (SIRPIDs): a common EEG phenomenon in the critically IIL. Epilepsia 2004;45:109-123.

4. Chong DJ, Hirsch LJ. Which EEG patterns warrant treatment in the critically IIl? Reviewing the evidence for treatment of periodic epileptiform discharges and related patterns. J Clin Neurophysiol 2005;22:79-91.

5. Hirsch LJ. Classification of EEG patterns in patients with impaired consciousness. Epilepsia 2011;52(Suppl8):S21-S24.

6. Chatrian G, Shaw C, Leffman H. The significance of periodic lateralized epileptiform discharges in EEG:an electrographic, clinical and pathological study. Electroencephalogr Clin Neurophysiol 1964;17:177-193.

7. Fisch BJ, Klass DW. The diagnostic specificity of triphasic wave patterns. Electroencephalogr Clin Neurophysiol 1988;70:1-8.
8. Hirsch LJ, Claassen J, Emerson RG. SRPIDs: stimulus-responsive pseudo-ictal discharges: a common and important EEG pattern in critically ill patients. Epilepsia 2002;43(suppl 7):45

9. Steriade M. Arousal: revisiting the reticular activating system. Science 1996;272:225-226.

10. Steriade M. Corticothalamic resonance, states of vigilance and mentation. Neuroscience 2000;101:243-276.

11. Steriade M. Synchronized activities of coupled oscillators in the cerebral cortex and thalamus at different levels of vigilance. Cereb Cortex 1997;7:583-604.

12. Murphy MM. SIRPIDs: a case study. Am.J. END Technol 2006;46:159-164

13. Fluri F, Balestra G, Christ M, Marsch S, Fuhr P, Rüegg S. Stimulusinduced rhythmic, periodic or ictal discharges (SIRPID's) exclusively elicited by stimulation of the ophthalmic Nerve. Clin Neurophysiology 2008;119:1934-1938.

14. RossettiAO.Creutzfeldt-Jakob disease: evolution from nonconvulsive status epilepticus, through SIRPIDs, to generalized periodic discharges. Clin Neurophysiol 2007;118:2533-2538. 
15. Koutroumanidis M,Tsatsou K, Bonakis A, Michael M,Tan SV. Stimulus-induced bilateral central periodic discharges, cortical myoclonus and arousal responses in mild reversible coma. Clin Neurophysiol 2008;119:2459-2464.

16. Ong C, Gilmore E, Claassen J, Brandon F, Mayer SA. Impact of prolonged periodic epileptiform discharges on coma prognosis. Neurocrit Care 2012;17:39-44.

17. Claassen J, Mayer SA, Kowalski RG, Emerson RG, Hirsch LJ. Detection of electrographic seizures with continuous EEG monitoring in critically ill patients. Neurology 2004; 62:1743-1748.
18. Zeiler SR, Turtzo LC, Kaplan PW. SPECT-negative SIRPIDs argues against treatment as seizures.J Clin Neurophysiol 2011;28:493-496.

19. Alvarez V, Oddo M, Rossetti AO. Stimulus-induced rhythmic, periodic or ictal discharges (SIRPIDs) in comatose survivors of cardiac arrest: Characteristics and prognostic value. Clin Neurophysiol 2013;124:204-208.

20. Anzelloti F, Franciotti R, Onofrj M. Stimulus aborted periodic epileptiform discharges: A case description and possible relationship with stimulus induced discharges. Epilepsy Res, 2013, http://dx.doi. org/10.1016/j.eplepsyres.2013.03.013.

21. Bauer G, Trinka E. Nonconvulsive status epilepticus and coma. Epilepsia 2010; 51:177-190. 